\title{
Gut Microbiota-Derived Inflammation-Related Serum Metabolites as Potential Biomarkers for Major Depressive Disorder
}

\author{
Shunjie Bai $\mathbb{D}^{1, *}$ \\ Jing $\mathrm{Xie}^{2, *}$ \\ Huili Bai ${ }^{3}$ \\ Tian $\operatorname{Tian}^{4}$ \\ Tao Zou ${ }^{5,6}$ \\ Jian-Jun Chen ${ }^{7}{ }^{7}$
}

'Department of Laboratory Medicine, The First Affiliated Hospital of Chongqing Medical University, Chongqing, People's Republic of China; ${ }^{2}$ Department of Endocrinology, the Fourth People's Hospital of Chongqing, Chongqing University Central Hospital, Chongqing, People's Republic of China; ${ }^{3}$ The Center for Clinical Molecular Medical Detection, The First Affiliated Hospital of Chongqing Medical University, Chongqing, People's Republic of China; ${ }^{4}$ Department of Neurology, the Affiliated Hospital of Guizhou Medical University, Guiyang, Guizhou Province, People's Republic of China; ${ }^{5}$ Department of Psychiatry, the Affiliated Hospital of Guizhou Medical University, Guiyang, Guizhou Province, People's Republic of China; ${ }^{6}$ Shanghai Key Laboratory of Forensic Medicine, Academy of Forensic Science, Shanghai, People's Republic of China; ${ }^{7}$ Institute of Life Sciences, Chongqing Medical University, Chongqing, People's Republic of China

*These authors contributed equally to this work

Correspondence: Jian-Jun Chen Institute of Life Sciences, Chongqing Medical University, I Yixueyuan Road, Yuzhong

District, Chongqing, 4000 16, People's

Republic of China

Email chenjianjun@cqmu.edu.cn

Tao Zou

Department of Psychiatry, The Affiliated Hospital of Guizhou Medical University, Yunyan District, Guiyang, Guizhou Province, 550004, People's Republic of China

Email zoutaozou@tom.com
Background: Although many works have been conducted to explore the biomarkers for diagnosing major depressive disorder (MDD), the widely accepted biomarkers are still not identified. Thus, the combined application of serum metabolomics and fecal microbial communities was used to identify gut microbiota-derived inflammation-related serum metabolites as potential biomarkers for MDD.

Methods: MDD patients and healthy controls (HCs) were included in this study. Both serum samples and fecal samples were collected. The liquid chromatography mass spectrometry (LC-MS) was used to detect the metabolites in serum samples, and the 16S rRNA gene sequencing was used to analyze the gut microbiota compositions in fecal samples.

Results: Totally, $60 \mathrm{MDD}$ patients and $60 \mathrm{HCs}$ were recruited. The 24 differential serum metabolites were identified, and 10 of these were inflammation-related metabolites. Three significantly affected inflammation-related pathways were identified using differential metabolites. The 17 differential genera were identified, and 14 of these genera belonged to phyla Firmicutes. Four significantly affected inflammation-related pathways were identified using differential genera. Five inflammation-related metabolites (LysoPC(16:0), deoxycholic acid, docosahexaenoic acid, taurocholic acid and LysoPC(20:0)) were identified as potential biomarkers. These potential biomarkers had significant correlations with genera belonged to phyla Firmicutes. The panel consisting of these biomarkers could effectively distinguish MDD patients from HCs with an area under the curve (AUC) of 0.95 in training set and 0.92 in testing set.

Conclusion: These findings suggested that the disturbance of phyla Firmicutes might be involved in the onset of depression by regulating host's inflammatory response, and these potential biomarkers could be useful for future investigating the objective methods for diagnosing MDD.

Keywords: major depressive disorder, gut microbiota, biomarkers, inflammation, metabolite

\section{Introduction}

Major depressive disorder (MDD) is a serious mental disorder affecting millions of people every year. ${ }^{1}$ It causes significant economic burden for individuals and society. However, the pathogenesis of MDD is still unclear; ${ }^{2-4}$ the mainstream view is that the molecular brain dysfunctions are mainly responsible for this disease. $^{5}$ Unfortunately, the current first-line antidepressant therapies developed according to these prevailing theories can only alleviate depressive symptoms in about half of MDD patients. ${ }^{6}$ Meanwhile, the clinicians still diagnose MDD 
according to the subjective identification of symptom clusters of patients, rather than using objective tests. Up to now, there are still no widely accepted biomarkers, although many potential biomarkers have been identified based on the existing theories. ${ }^{7-10}$ These phenomena indicate that the existing hypotheses cannot comprehensively reflect the heterogeneous pathophysiological mechanisms of MDD. Therefore, it is urgently needed to explore novel molecular mechanisms of MDD.

Nowadays, mounting evidence suggests that gut microbiota has a vital role in the onset of neuropsychiatric disorders. ${ }^{11,12}$ It can interact with the physiological processes of host through its metabolic products, such as short-chain fatty acids (SCFAs). ${ }^{13,14}$ Many studies have shown that the disturbance of gut microbiota has become an important factor in the pathogenesis of depression. ${ }^{15,16}$ Using nonhuman primate model of depression, researchers found that gut microbiota might participate in the onset of depression through regulating the glycerophospholipid metabolism. ${ }^{15}$ Using a mouse model of depression, we observed the close relationships between the disordered gut microbiota and hypothalamus neurotransmitters. ${ }^{16}$ Meanwhile, our studies in humans also found that there were significant differences on gut microbiota compositions between MDD patients and healthy controls (HCs). ${ }^{17,18}$ The disturbance of gut microbiota in depressed patients was also observed in other studies. ${ }^{19,20}$ These findings demonstrate that further exploring the role of gut microbiota in MDD can provide some novel insights in revealing the pathogenesis of this disease.

Gut microbiota can affect the health of host by regulating its inflammatory level. Gut microbiota-derived metabolites may be involved in the pathogenesis of immune-related inflammatory diseases by leading to the release of many inflammation-related serum markers, such as interleukins (ILs), C-reactive protein (CRP) and tumor necrosis factor (TNF). ${ }^{21,22}$ Previous studies reported that inflammation had a close relationship with the pathogenesis of depression, and individuals with inflammatory disorders might have an increased incidence of depression. ${ }^{23,24}$ Zheng et al found five significantly changed gut microbiota-related urinary metabolites in MDD patients, and these metabolites had some relationships with inflammatory response. ${ }^{25}$ Our previous study found that plasma metabolic abnormalities in lipid and amino acid metabolism might be implicated in the occurrence of suicidal behaviors in MDD patients. ${ }^{26}$ Both gut microbiota and inflammatory response have close relationships with lipid metabolites. Meanwhile, we also found that the decreased level of alpha 1-antitrypsin (AAT) might lead to inflammatory response disorders, and ultimately lead to the occurrence of suicidal ideation in MDD patients. ${ }^{27}$ The inflammatory response and immune system represent the major component of communications between gut microbiota and brain functions. Accumulating evidence supports the role of gut microbiota in regulating the host metabolic and immune functions. ${ }^{28,29}$ Therefore, we conducted this study to further explore the differences of gut microbiota and serum metabolites between MDD patients and HCs. Our findings will advance our understanding of how gut microbiota contributes to the onset of depression.

\section{Patients and Methods MDD Patients and HCs Enrolment}

This study was approved by the Ethical Committee of Chongqing Medical University (Approved No. 20200320). All the included participants provided the written informed consents before samples collecting. This study was conducted in accordance with the Declaration of Helsinki. Two experienced psychiatrists were in charge of participants' recruitment using the [Diagnostic and Statistical Manual of Mental Disorders, 4th edition] criteria (DSM-IV). MDD patients met the following criteria were included in this study: i) 17-item Hamilton Depression Rating Scale (HDRS) was used to assess the depression severity, and the candidates with HDRS scores $>17$ were included into this study; ii) without any pre-existing mental disorders, and without other DSM-IV Axis I/II disorders; iii) we did not recruit women who were pregnant or within the first year after delivery; iv) without any illicit drug use or alcohol abuse; and v) did not receive any antidepressive treatments in one month prior to sample collections. Meanwhile, two experienced psychiatrists applied DSM-IV to assess the mental state of HCs, and used the physical examination records to carefully check the other information of HCs. Finally, HCs without DSM-IV Axis I/II disorders, systemic medical illness, illicit drug use and alcohol abuse were recruited from the Medical Examination Center of our hospital. The detailed information is described in Table 1.

\section{Gut Microbiota Compositions Detection}

The gut microbiota analysis was conducted according to the standard protocols by Majorbio Bio-Pharm Technology Co. Ltd. (Shanghai, China). The raw 16S rRNA gene sequencing reads were demultiplexed, quality-filtered 
Table I Demographic Data of Included Subjects in This Study

\begin{tabular}{|l|l|l|l|}
\hline Variables & HCs & $\begin{array}{l}\text { MDD } \\
\text { Patients }\end{array}$ & P-value \\
\hline Number & 60 & 60 & - \\
Age & $35.13(15.79)$ & $35.62(17.10)$ & 0.87 \\
Sex $(\mathrm{F} / \mathrm{M})$ & $36 / 24$ & $39 / 21$ & 0.57 \\
BMI $\left(\mathrm{kg} / \mathrm{m}^{2}\right)$ & $21.19(4.29)$ & $20.90(2.30)$ & 0.63 \\
Married $(\mathrm{Y} / \mathrm{N})$ & $32 / 28$ & $36 / 24$ & 0.46 \\
Family history of PI $(\mathrm{Y} / \mathrm{N})$ & $4 / 56$ & $6 / 54$ & $0.5 \mathrm{I}$ \\
HDRS scores & $0.67(0.93)$ & $25.3(6.0 \mathrm{I})$ & $<0.0000 \mathrm{I}$ \\
\hline
\end{tabular}

Abbreviations: MDD, major depressive disorder; $\mathrm{HCs}$, healthy controls; $\mathrm{Y}$, yes; $\mathrm{N}$, no; HDRS, Hamilton Depression Rating Scale; F, female; M, male; BMI, body mass index, Pl, psychiatric illness.

with Trimmomatic, and merged with FLASH according to the following criteria: i) set 50-bp sliding window; if the average quality score was less than 20 in the window, the back-end base is cut off from the window; the reads below 50-bp were filtered to remove the n-base reads; ii) according to the overlap relationships between reads, the paired reads were merged into one sequence, and the minimum overlap length was $10 \mathrm{bp}$; iii) the maximum allowable mismatch ratio of overlap region of splicing sequence was 0.2 ; and iv) according to the barcodes and primers at both ends of the sequence, the samples were distinguished, and the sequence direction was adjusted. The number of allowed mismatches of barcodes was 0 , and the maximum number of primer mismatches was 2. After cleaning up, the remaining sequences were assigned to operational taxonomic units (OTUs) with $97 \%$ similarity cutoff. The taxonomy of each OTU was classified using RDP reference database. Finally, we used these taxonomies to construct the summaries of the taxonomic distributions of OTUs. The taxonomic distributions of OTUs were used to calculate the relative abundances of gut microbiota at different levels.

\section{Serum Metabolomics Detection}

Firstly, $50 \mu \mathrm{L}$ serum and $300 \mu \mathrm{L}$ of acetonitrile were vortex-mixed. After centrifugation $\left(4^{\circ} \mathrm{C}, 15\right.$ minutes, $14,000 \mathrm{rpm})$, we transferred the supernatant into a new tube and then conducted vacuum drying. Secondly, we added $20 \mu \mathrm{L}$ acetonitrile into the obtained residue and conducted vortex with $80 \mu \mathrm{L}$ water. After centrifugation $\left(4^{\circ} \mathrm{C}, 15\right.$ minutes, $\left.14,000 \mathrm{rpm}\right)$, we transferred $80 \mu \mathrm{L}$ supernatant into a glass vial for later liquid chromatography mass spectrometry (LC-MS) analysis. An ACQUITY I Class UPLC system coupled to a Waters G2-S QTOF system, with a Waters ACQUITY UPLC HSS T3 column and a Waters ACQUITY UPLC HSS T3 VanGuard precolumn was used to conduct the metabolomic analysis of the sample extracts. We added $5 \mu \mathrm{L}$ of extract into this system for metabolomic fingerprinting.

\section{Statistical Analysis}

All the statistical analyses were conducted using SPSS 19.0 and $\mathrm{R}$ 3.6.0. The principal-coordinate analysis (PCoA) was used to visualize the discrimination between the two groups. The linear discriminant-analysis effect size (LEfSe) was then conducted to identify the differential bacterial taxa responsible for the discrimination between the two groups. The orthogonal partial least-squares discriminant analysis (OPLS-DA) was performed to explore the significant differences on serum metabolites between the two groups. The Pearson correlation analysis was conducted to evaluate the correlations between the differential bacterial taxa and the differential metabolites. Finally, potential biomarkers from these differential metabolites were identified using Random Forest analysis. The receiver operating characteristic (ROC) curve analysis was further performed to assess the diagnostic performance of the identified potential biomarkers. ${ }^{30-33}$ The area under the curve (AUC) was the evaluation index. In general, an AUC of 0.5 to $07,0.7$ to $0.8,0.8$ to 0.9 and 0.9 to 1.0 were considered no discrimination, acceptable, excellent, and outstanding, respectively. ${ }^{34}$ All statistical analyses were two-tailed and the significance level was set at p-value $<0.05$.

\section{Results \\ Gut Microbial Compositions in MDD Patients and HCs}

Using 16s rRNA gene sequencing, a total of $6,414,608$ high-quality reads across 120 samples were obtained. The average length in samples was 414 base pairs (bp). We clustered these reads into 1325 OTUs at $97 \%$ sequence similarity. There were 1034 OTUs in both groups, while 128 OTUs and 163 OTUs were unique to MDD patients and HCs, respectively (Figure 1). These OTUs mainly belonged to four phyla: Firmicutes (58.11\%), Bacteroidota (22.11\%), Proteobacteria $(7.02 \%)$ and Actinobacteriota $(6.49 \%)$. The within-sample $(\alpha)$ phylogenetic diversity analysis showed that no significant difference was found between the two groups on alpha diversity (Simpson, $\mathrm{p}=0.25$; Shannon, $\mathrm{p}=0.18$; chao, $\mathrm{p}=0.62$ and phylogenetic diversity, $\mathrm{p}=0.42$; Figure 1 ). 

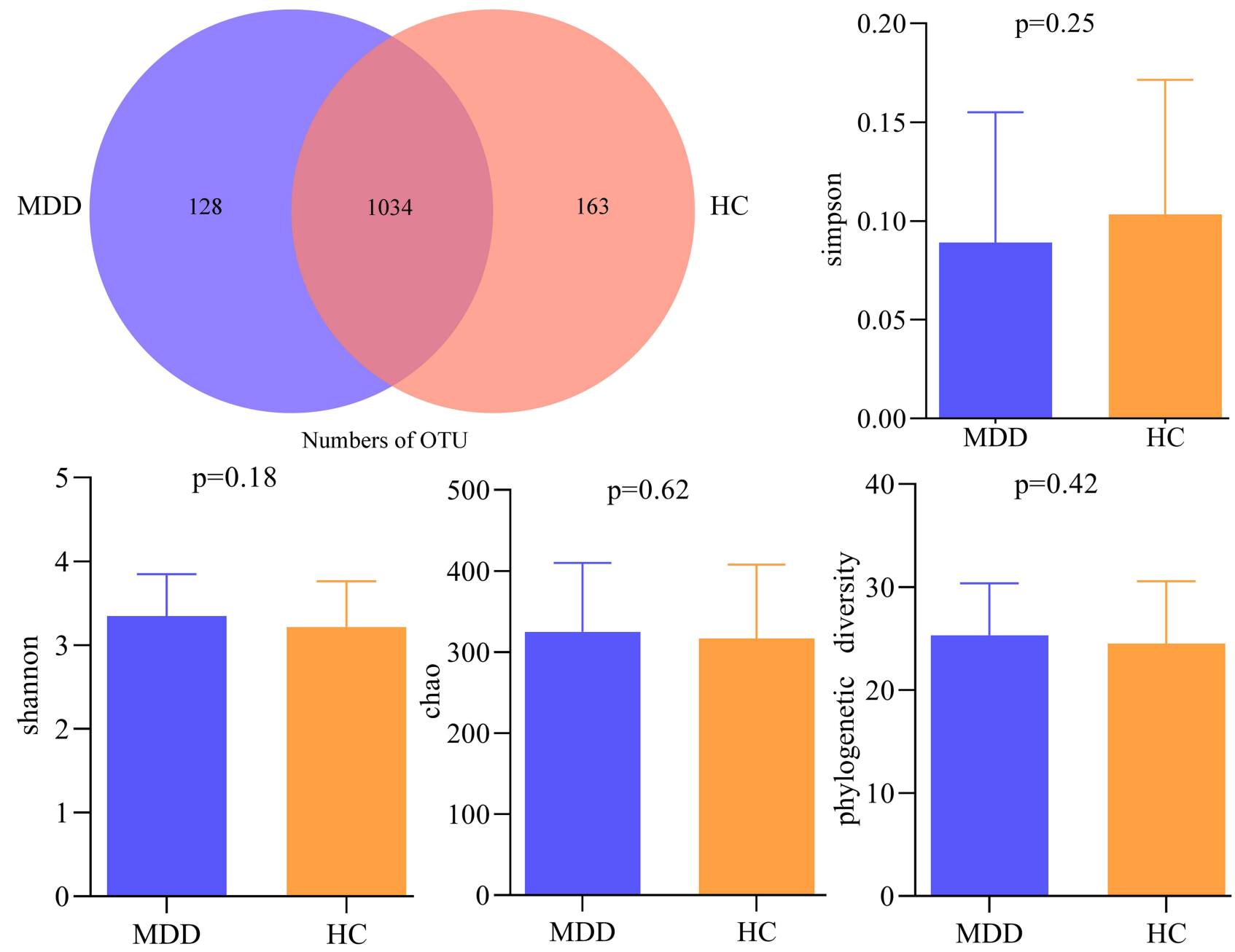

Figure I Gut microbial compositions and $\alpha$-diversity between the two groups.

Abbreviations: MDD, major depressive disorder; HC, healthy controls; OTU, operational taxonomic unit.

\section{Differential Gut Microbiota in MDD}

\section{Patients}

Meanwhile, we conducted the $\beta$-diversity analysis to find out whether the gut microbiota compositions were substantially different in MDD patients compared to HCs. The PCoA results showed that there were significant differences on gut microbial compositions between MDD patients and HCs (Figure 2A, $\mathrm{p}=0.002$ ). The dominant bacteria taxa on Family level in MDD patient and HCs were Lachnospiraceae $(27.79 \%$ vs $26.93 \%)$, Ruminococcaceae $(16.78 \%$ vs $17.04 \%)$, Bacteroidaceae (15.99\% vs $14.95 \%)$, Prevotellaceae (7.75\% vs 9.91\%), Selenomonadaceae $(4.07 \%$ vs $5.20 \%$ ), Bifidobacteriaceae (3.91\% vs $4.22 \%)$ and Enterobacteriaceae $(3.78 \%$ vs $5.26 \%$ ) (Figure 2B). The percent of community abundance on family level in each sample is described in Figure 2C. Moreover, using LEfSe, we identified 17 differential genera responsible for the discrimination between the two groups: Lactobacillus, Colidextribacter, Holdemania, norank_f_Lachnospiraceae, GCA-900066575, Lachno clostridium, Eubacterium_ventriosum_group, Candidatus_ Soleaferrea, Faecalitalea, CAG-56, Lachnospiraceae_ NK4A136_group, Lachnospira, Clostridium_innocuum group, unclassified_f_Lachnospiraceae, Collinsella, Parabacteroides, and Klebsiella. The former 14 differential genera belonged to phyla Firmicutes.

\section{Differential Serum Metabolites in MDD Patients}

OPLS-DA results showed that the MDD patients and HCs were obviously separated with little overlap (Figure 3A). The positive values of the two model evaluation indexes $\left(\mathrm{R}^{2}=76 \%, \mathrm{Q}^{2}=63 \%\right)$ indicated that the built model was stable. Meanwhile, the 399-item permutation test also 


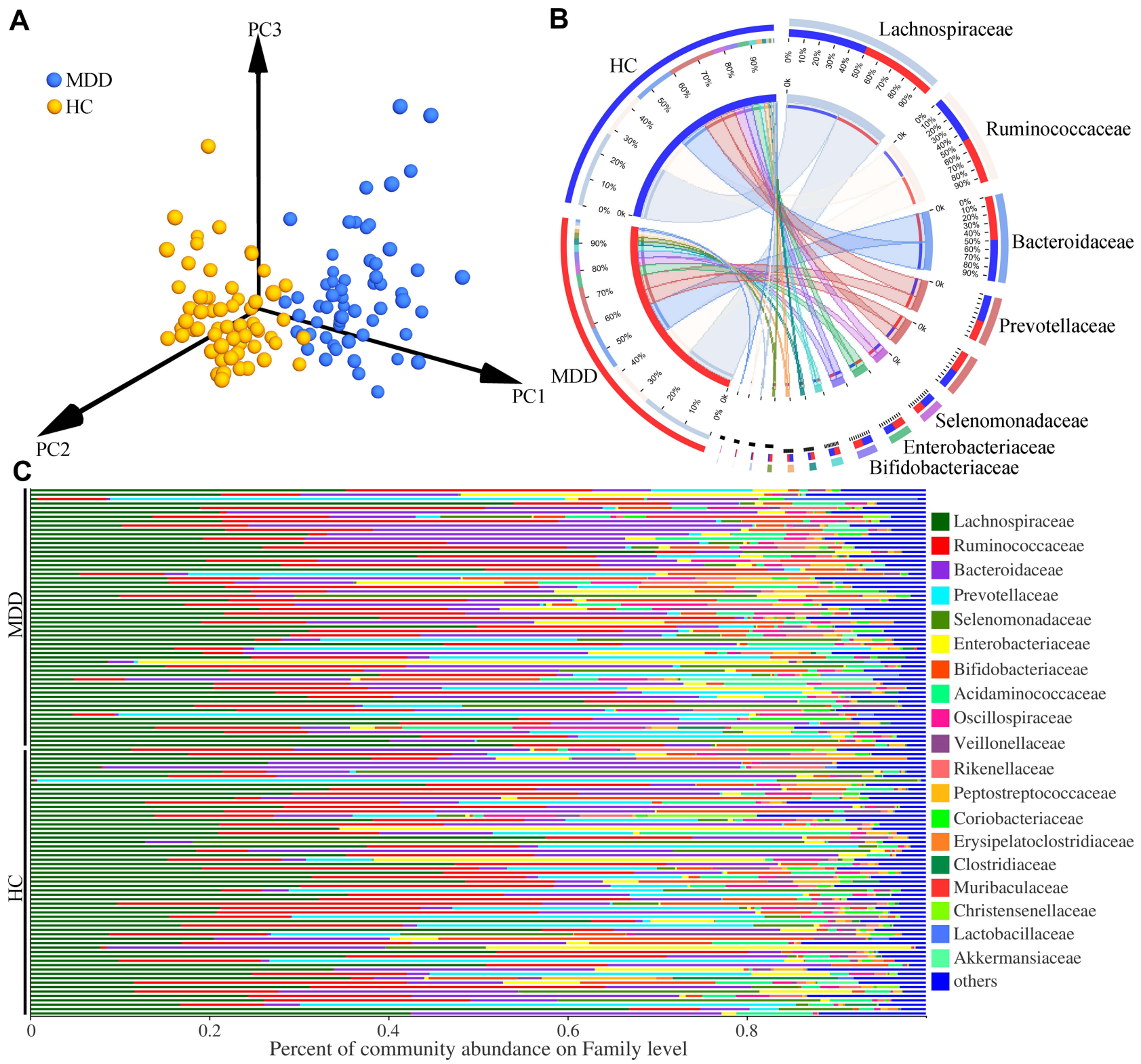

Figure 2 Differential gut microbiota compositions between the two groups. (A) The significant differences on gut microbial compositions between the two groups were identified by the results of PCoA; (B) the dominant bacteria taxa on Family level in MDD patients and HCs; (C) the percent of community abundance on Family level in each sample.

Abbreviations: MDD, major depressive disorder; HC, healthy controls.

showed that our model was valid and not over-fitted, because the corresponding permutated values of $\mathrm{R}^{2}$ and $\mathrm{Q}^{2}$ were obviously lower compared to their original values (Figure 3B). These results demonstrated that there was a divergent metabolic phenotype between MDD patients and HCs. By analyzing the loading coefficient plot of OPLS-DA model, we identified 24 metabolites with a correlation coefficient $>0.254$ (equivalent to a $p$-value of less than 0.05 ) as the differential metabolites responsible for the discrimination between the two groups.
As compared to HCs, MDD patients were characterized by higher levels of Uridine triphosphate, N-(2RHydroxytricosanoyl)-2S-amino-1,3S,4R-octadecanetriol (N4R), Benzoic acid, 1-Heptadecanoyl-glycero-3-phosphocholine [LysoPC(17:0)], N-[(15Z)-Tetracosenoyl]sphing4-enine 1-phosphate [CerP(d18:1/24:1(15Z))], 1-Palmitoyl-2-oleoyl-gpe $\quad[\mathrm{PE}(16: 0 / 18: 1(9 Z))], \quad$ 5-Hexatri acontanone, N-(13Z-Docosenoyl)-sphinganine-1-phosphocholine $[\mathrm{SM}(\mathrm{d} 18: 0 / 22: 1(13 \mathrm{Z}))], \quad$ 1-Eicosadienoylglycero phosphocholine [GPC(20:2/0:0)] and 1-Palmitoyl- 


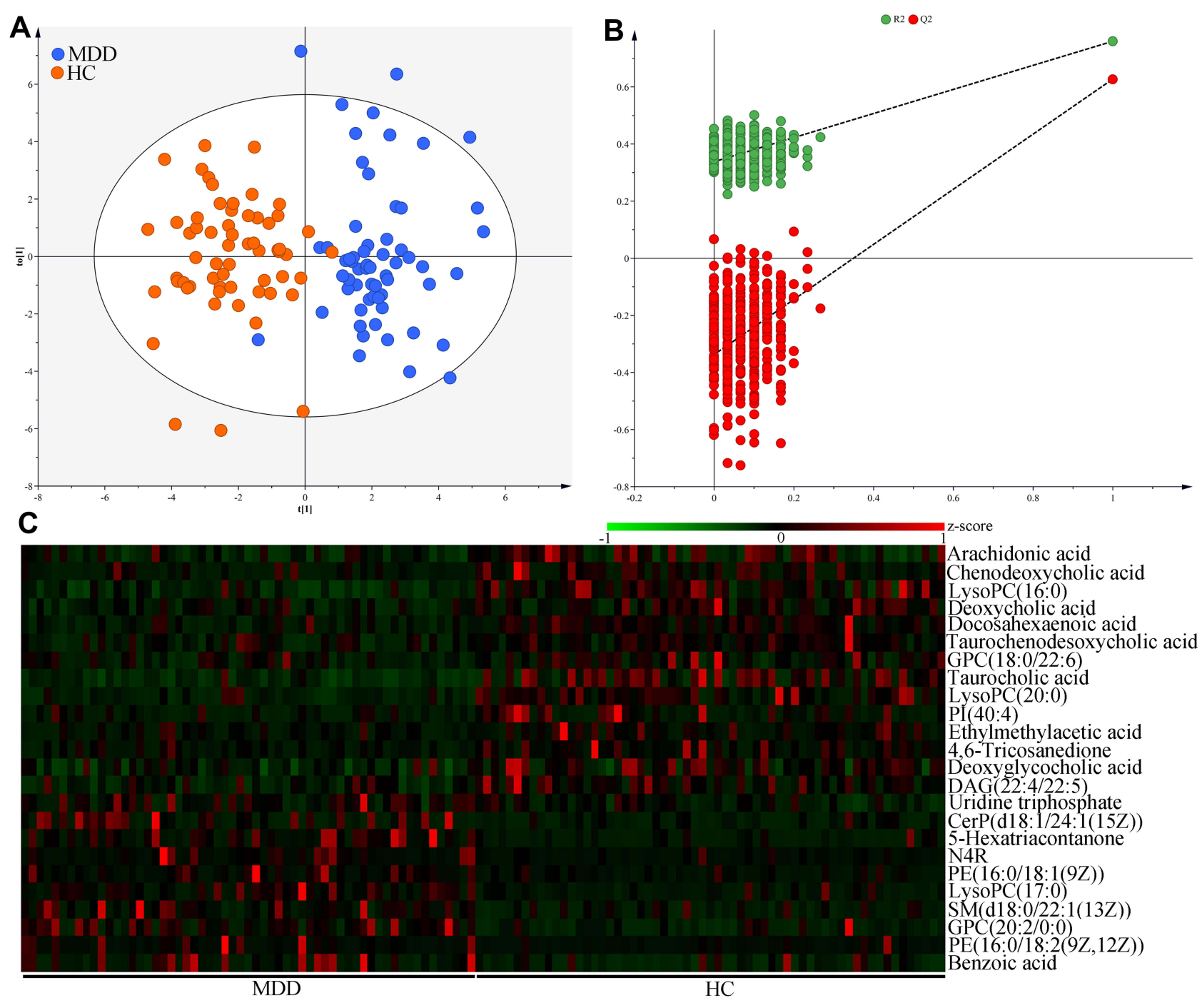

Figure 3 Metabolomic analysis of serum samples from MDD patients and HCs. (A) The divergent serum metabolic phenotype was observed between the two groups; (B) the results of 399-item permutation test indicated that the discriminative model was robust; (C) the heatmap of the identified 24 differential serum metabolites.

Abbreviations: MDD, major depressive disorder; HC, healthy controls. PI(40:4), Phosphatidylinositol(40:4); GPC(20:2/0:0), I-Eicosadienoylglycerophosphocholine; GPC (I8:0/22:6), I-Stearoyl-2-docosahexaenoyl-sn-glycero-3-phosphocholine; PE(I6:0/I8:2(9Z,I2Z)), I-Palmitoyl-2-linoleoyl-gpe; CerP(dI8:I/24:I(I5Z)), N-[(I5Z)Tetracosenoyl]sphing-4-enine I-phosphate; N4R, N-(2R-Hydroxytricosanoyl)-2S-amino-I,3S,4R-octadecanetriol; LysoPC(I7:0), I-Heptadecanoyl-glycero-3-phosphocholine; LysoPC(I6:0), I-16:0-Lysophosphatidylcholine; SM(dI8:0/22:I(I3Z)), N-(I3Z-Docosenoyl)-sphinganine-I-phosphocholine; DAG(22:4/22:5), I-Adrenoyl-2-docosapentaenoyl-sn-glycerol; LysoPC(20:0), I-Arachidonyl-glycero-3-phosphocholine; PE(I6:0/I8:I(9Z)), I-Palmitoyl-2-oleoyl-gpe.

2-linoleoyl-gpe [PE(16:0/18:2(9Z,12Z))], along with lower levels of Arachidonic acid, Chenodeoxycholic acid, 116:0-Lysophosphatidylcholine

[LysoPC(16:0)],

Deoxycholic acid, Docosahexaenoic acid, 1-Stearoyl-2-docosahexaenoyl-sn-glycero-3-phosphocholine [GPC(18:0/ 22:6)], Taurocholic acid, Taurochenodeoxycholic acid, 1-Arachidonyl-glycero-3-phosphocholine [LysoPC(20:0)], Phosphatidylinositol(40:4)[PI(40:4)], Ethylmethylacetic acid, 4.6-Tricosanedione, Deoxyglycocholic acid and 1-Adrenoyl-2-docosapentaenoyl-sn-glycerol [DAG (22:4/
22:5)]. There were ten inflammation-related metabolites: Arachidonic acid, Chenodeoxycholic acid, LysoPC(16:0), Deoxycholic acid, Docosahexaenoic acid, Taurochenodeoxycholic acid, GPC(18:0/22:6), Taurocholic acid, LysoPC(20:0), PI(40:4), Ethylmethylacetic acid, 4.6-Tricosanedione and Deoxyglycocholic acid. Hierarchical clustering heat map of these differential metabolites showed a consistent clustering pattern within individual groups (Figure 3C). The detailed information of these differential metabolites is described in Table 2. 
Table 2 Differential Metabolites Between MDD Patients and HCs

\begin{tabular}{|c|c|c|c|c|}
\hline Metabolites & $\mathbf{R}^{\mathbf{a}}$ & $\mathbf{P}^{\mathbf{b}}$ & $\mathbf{A P}^{\mathbf{c}}$ & $F^{c}$ \\
\hline Arachidonic acid & -0.503 & 4.94E-05 & $4.82 \mathrm{E}-04$ & 1.89 \\
\hline Chenodeoxycholic acid & -0.365 & I.60E-03 & $6.59 \mathrm{E}-03$ & 2.36 \\
\hline LysoPC (16:0) & -0.474 & $2.02 \mathrm{E}-09$ & $7.90 \mathrm{E}-08$ & 2.45 \\
\hline Deoxycholic acid & -0.378 & 7.59E-05 & $6.58 \mathrm{E}-04$ & 1.53 \\
\hline Docosahexaenoic acid & -0.724 & I.87E-07 & 4.87E-06 & 2.03 \\
\hline Taurochenodeoxycholic acid & -0.322 & $8.00 \mathrm{E}-04$ & 3.67E-03 & 1.51 \\
\hline GPC $(18: 0 / 22: 6)$ & -0.262 & 8.67E-03 & $2.05 \mathrm{E}-02$ & 1.52 \\
\hline Taurocholic acid & -0.728 & I.3IE-II & $1.02 \mathrm{E}-09$ & 3.46 \\
\hline LysoPC(20:0) & -0.368 & $9.43 \mathrm{E}-07$ & I.84E-05 & 2.27 \\
\hline $\mathrm{PI}(40: 4)$ & -0.286 & $2.87 \mathrm{E}-03$ & $9.34 \mathrm{E}-03$ & 2.55 \\
\hline Ethylmethylacetic acid & -0.361 & 7.7IE-03 & 2.07E-02 & 1.95 \\
\hline 4,6-Tricosanedione & -0.298 & I.67E-03 & 6.49E-03 & 2.21 \\
\hline Deoxyglycocholic acid & -0.315 & $3.36 \mathrm{E}-05$ & $3.75 \mathrm{E}-04$ & 1.67 \\
\hline DAG(22:4/22:5) & -0.276 & I.72E-04 & I. $12 \mathrm{E}-03$ & 1.68 \\
\hline Uridine triphosphate & 0.417 & $6.00 \mathrm{E}-04$ & $3.12 \mathrm{E}-03$ & 0.62 \\
\hline CerP(dI8:I/24:I(I5Z)) & 0.386 & $7.62 \mathrm{E}-05$ & $5.95 \mathrm{E}-04$ & 0.28 \\
\hline 5-Hexatriacontanone & 0.396 & 8.09E-06 & $1.26 \mathrm{E}-04$ & 0.19 \\
\hline N4R & 0.372 & 2.IIE-03 & 7.47E-03 & 0.26 \\
\hline $\operatorname{PE}(16: 0 / 18: 1(9 Z))$ & 0.326 & $3.40 \mathrm{E}-03$ & $1.02 \mathrm{E}-02$ & 0.40 \\
\hline LysoPC(I7:0) & 0.337 & I.I2E-04 & 7.96E-04 & 0.41 \\
\hline SM(dI8:0/22:I(I3Z)) & 0.339 & $7.69 \mathrm{E}-04$ & $3.75 \mathrm{E}-03$ & 0.45 \\
\hline $\operatorname{GPC}(20: 2 / 0: 0)$ & 0.436 & $3.35 \mathrm{E}-05$ & 4.36E-04 & 0.50 \\
\hline $\mathrm{PE}(16: 0 / 18: 2(9 Z, 12 Z))$ & 0.406 & $2.39 \mathrm{E}-02$ & 4.44E-02 & 0.34 \\
\hline Benzoic acid & 0.271 & $8.90 \mathrm{E}-04$ & $3.86 \mathrm{E}-03$ & 0.49 \\
\hline
\end{tabular}

Notes: ${ }^{a}$ Negative and positive value of correlation coefficient indicated significantly lower and higher, respectively, levels in MDD patients. ${ }^{\mathrm{b}} \mathrm{p}$-values from the non-parametric Mann-Whitney U-test. 'Adjusted $p$-values using Benjamini and Hochberg False Discovery Rate. ${ }^{d}>I$ and $<I$ indicated significantly lower and higher, respectively, levels in MDD patients.

Abbreviations: PI(40:4), Phosphatidylinositol(40:4); GPC(20:2/0:0), I-Eicosadienoylglycerophosphocholine; GPC(I8:0/22:6), I-Stearoyl-2-docosahexaenoyl-sn-glycero -3-phosphocholine; PE(I6:0/I8:2(9Z,I2Z)), I-Palmitoyl-2-linoleoyl-gpe; CerP(dI8:I/24:I(I5Z)), N-[(I5Z)-Tetracosenoyl]sphing-4-enine I-phosphate; N4R, N-(2RHydroxytricosanoyl)-2S-amino-I,3S,4R-octadecanetriol; LysoPC(I7:0), I-Heptadecanoyl-glycero-3-phosphocholine; LysoPC(I6:0), I-16:0-Lysophosphatidylcholine; SM (d I8:0/22: I ( I3Z)), N-(I3Z-Docosenoyl)-sphinganine-I-phosphocholine; DAG(22:4/22:5), I-Adrenoyl-2-docosapentaenoyl-sn-glycerol; LysoPC(20:0), I-Arachidonyl-glycero -3-phosphocholine; PE(I6:0/I8:I(9Z)), I-Palmitoyl-2-oleoyl-gpe; MDD, major depressive disorder; HCs, healthy controls; AP, adjusted p-value; FC, fold change.

\section{Pathway Analysis of Differential Metabolites and Genera}

The online software MetaboAnalyst 5.0 was used to analyze the biological functions of differential metabolites. The results showed that there were three inflammationrelated pathways in which these differential metabolites were mainly involved: Glycerophospholipid metabolism $(\mathrm{p}=0.004$, impact $=0.22)$, Bile acid metabolism $(\mathrm{p}=0.009$, impact $=0.02)$ and Arachidonic acid metabolism $(\mathrm{p}=0.045$, impact $=0.31$ ) (Figure 4A). Meanwhile, the PICRUSt was used to predict the biological functions of differential genera. The results showed that there were four inflammation-related pathways in which these differential genera were mainly involved: Glycerophospholipid metabolism $(\mathrm{p}=0.038)$, Bile acid metabolism $(\mathrm{p}=0.021)$, Nucleotide binding oligomerization domain (NOD)-like receptor signaling pathway $(\mathrm{p}=0.030)$, and Retinoic acid-inducible gene I (RIG-I)-like receptor signaling pathway $(\mathrm{p}=0.024)$ (Figure 4B).

\section{Potential Biomarkers for Diagnosing MDD}

To identify potential serum biomarkers for diagnosing MDD, the included subjects were randomly assigned into the training set (40 MDD patients and $40 \mathrm{HCs}$ ) and testing set (20 MDD patients and $20 \mathrm{HCs}$ ). Firstly, we used the training set to find out the potential biomarkers. The Random Forest analysis indicated that the most significant deviations on serum metabolites between MDD patients and HCs could be described using five inflammationrelated metabolites: LysoPC(16:0), Deoxycholic acid, Docosahexaenoic acid, Taurocholic acid and LysoPC (20:0). The ROC curve analysis showed that the panel consisting of these five metabolites could yield an AUC 

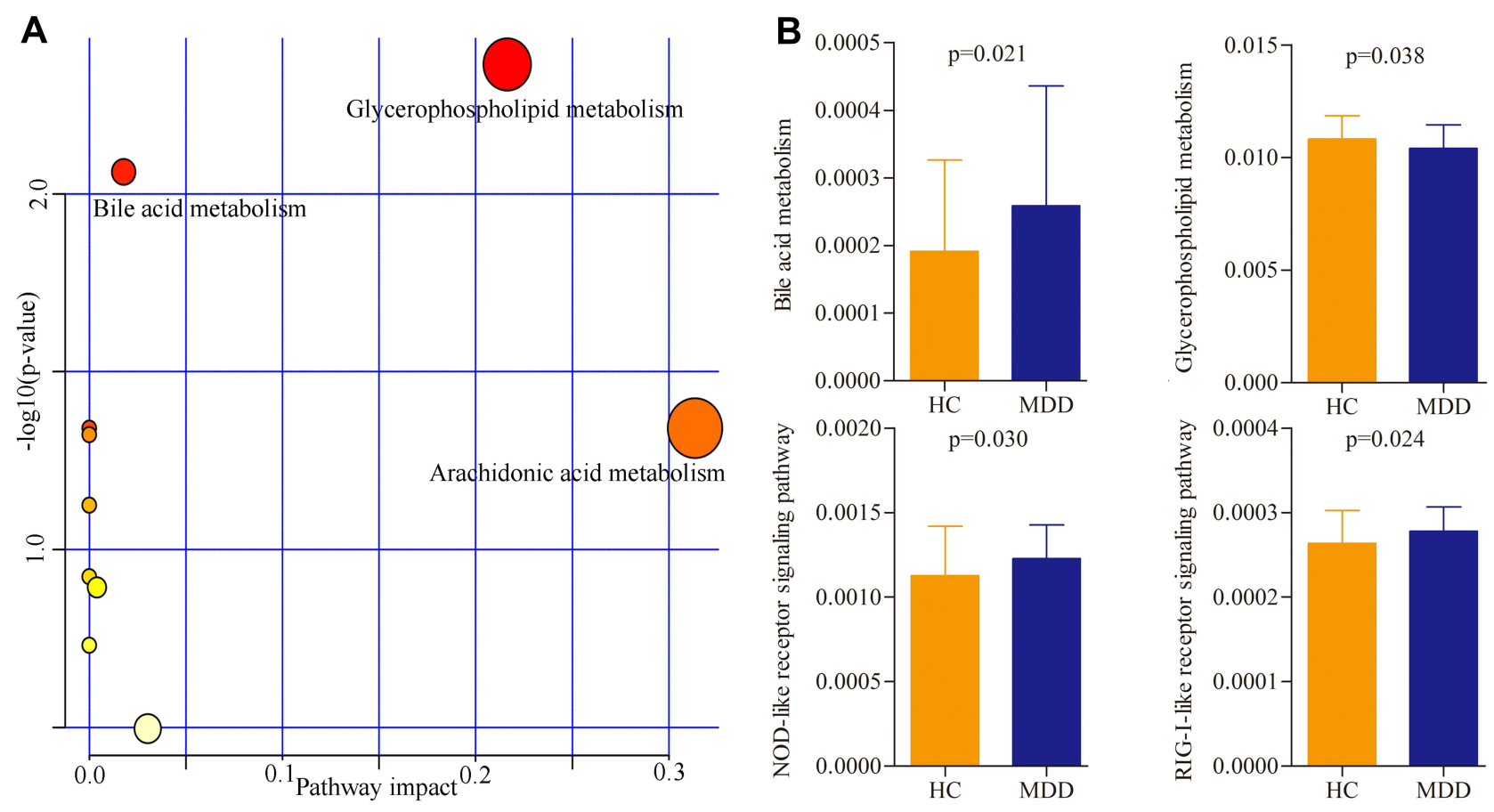

Figure 4 Biological functions of differential serum metabolites and genera. (A) Three significantly affected pathways that these differential serum metabolites were mainly involved in; (B) four significantly disturbed pathways that these differential genera were mainly involved in.

Abbreviations: MDD, major depressive disorder; HC, healthy controls.

of 0.95 (Figure 5A). Secondly, we used the testing set to validate the diagnostic performance of this panel, and the results showed that the panel could yield an AUC of 0.92 (Figure 5B). These results suggested that these five inflammation-related metabolites held the promise as the potential biomarkers for diagnosing MDD.

\section{Correlations Between Differential Metabolites and Genera}

In order to determine the possible relationships between gut microbiota and serum metabolites, the correlation analysis of differential metabolites and genera was conducted here. As shown in Figure 6, there were significant correlations between 11 differential genera (including 10 genera under phyla Firmicutes) and 15 differential serum metabolites (including 6 inflammation-related metabolites). The norank_f_Lachnospiraceae was significantly correlated with five metabolites (including two potential biomarkers), the Lachnospira was significantly correlated with four metabolites (including three potential biomarkers), the Faecalitalea was significantly correlated with three metabolites (including two potential biomarkers) and the Eubacterium_ventriosum_group was significantly correlated with one potential biomarkers. These results indicated that there were close relationships between the disordered phyla Firmicutes and host's inflammatory response.

\section{Discussion}

In this study, we found that there were significant differences on gut microbiota compositions and serum metabolites in MDD patients compared to HCs. In total, 24 differential serum metabolites were identified, and a panel consisting of $\operatorname{LysoPC}(16: 0)$, Deoxycholic acid, Docosahexaenoic acid, Taurocholic acid and LysoPC (20:0) was found to be effective in separating MDD patients from HCs. Meanwhile, 17 differential genera responsible for the discrimination between the two groups were identified. Furthermore, we observed the significantly positive correlations between the disturbance of phyla Firmicutes and these identified potential biomarkers. Considering the important role of gut microbiota in the onset of depression, our findings suggested that our identified biomarker panel might be a "good" classifier of MDD patients and HCs.

Glycerophospholipid metabolism has a close relationship with inflammatory response. For example, Lysophosphatidic acid (LPA) plays both anti- and proinflammatory roles in inflammatory lung diseases. ${ }^{35}$ Bile acids are signaling molecules, which could coordinately 

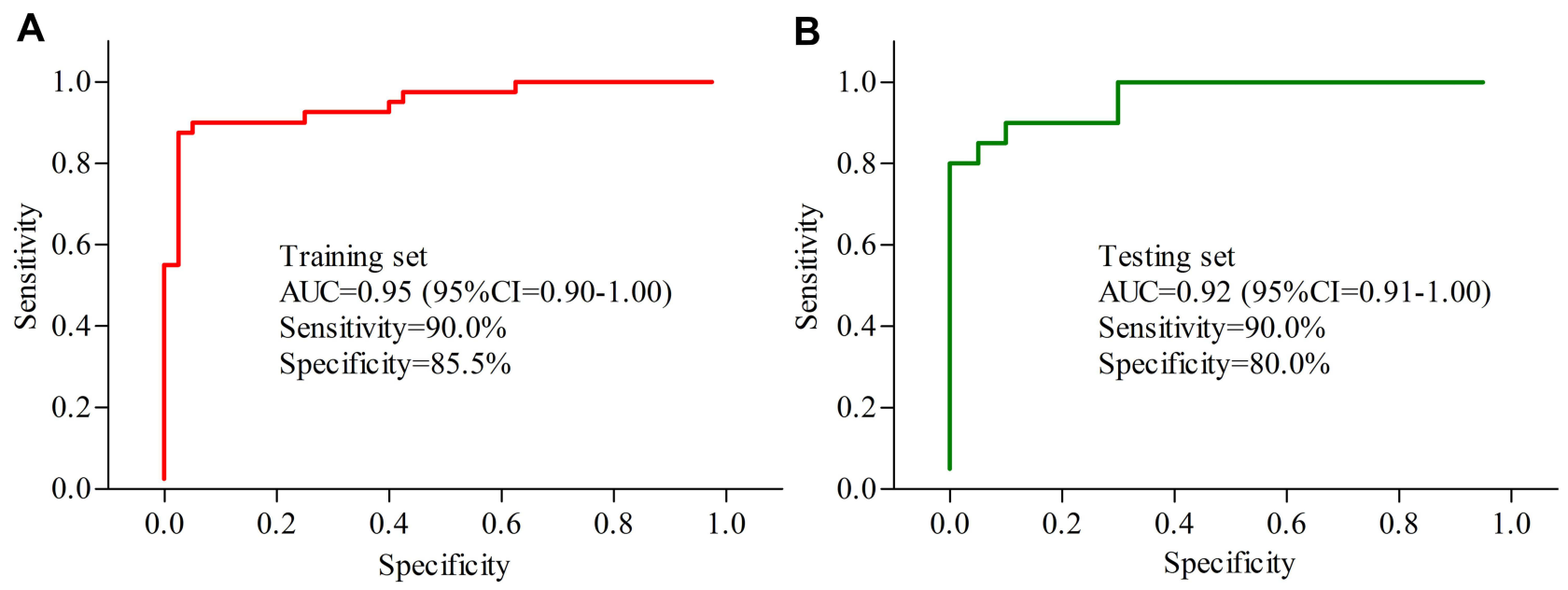

Figure 5 Diagnostic performance of the biomarker panel assessment. (A) The biomarker panel yielded an area under the curve (AUC) of 0.95 in the training set; (B) the AUC of the biomarker panel in the testing set was 0.92 .

Abbreviation: $\mathrm{Cl}$, confidence interval.

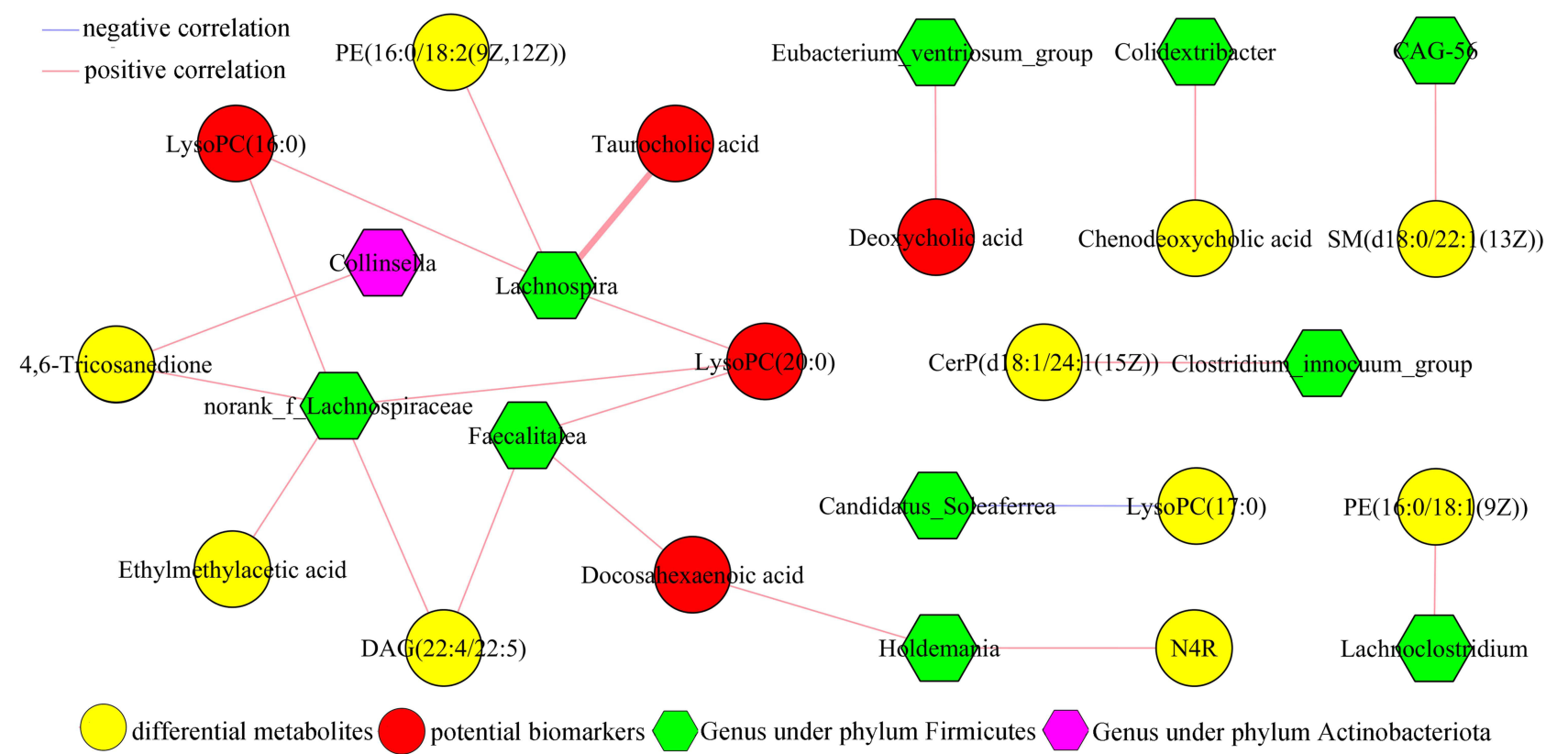

Figure 6 Correlations analysis of differential serum metabolites and genera.

Abbreviations: LysoPC(16:0), I-I6:0-Lysophosphatidylcholine; PE(I6:0/I8:2(9Z,I2Z)), I-Palmitoyl-2-linoleoyl-gPe; LysoPC(20:0), I-Arachidonyl-glycero-3-phosphocholine; LysoPC(I7:0), I-Heptadecanoyl-glycero-3-phosphocholine; CerP(dI8:I/24:I(I5Z)), N-[(I5Z)-Tetracosenoyl]sphing-4-enine I-phosphate; DAG(22:4/22:5), I-Adrenoyl2-docosapentaenoyl-sn-glycerol; PE(I6:0/I8:I(9Z)), I-Palmitoyl-2-oleoyl-gpe; N4R, N-(2R-Hydroxytricosanoyl)-2S-amino-I,3S,4R-octadecanetriol; SM(dI8:0/22:I(I3Z)), $\mathrm{N}$-(I3Z-Docosenoyl)-sphinganine-I-phosphocholine.

regulate inflammation and metabolism via Takeda G protein-coupled receptor 5 (TGR5) and nuclear farnesoid X receptor (FXR). ${ }^{36}$ Some metabolic products of arachidonic acid are the important inflammatory mediators, which may be important in leucocyte-mediated aspects of chronic inflammation. ${ }^{37}$ NOD-like receptors are the vital regulators in inflammation-associated angiogenesis, tumorigenesis, and cancer cell stemness and chemoresistance. ${ }^{38,39}$ Activated RIG-I-like receptors can activate the production of inflammatory cytokines and the type I/III interferons. ${ }^{40}$ In this study, the significantly changed serum metabolites and gut microbiota in MDD patients were significantly involved in these pathways. These results further demonstrated the close relationships between gut microbiota, inflammation and depression. 
Gut microbiota plays a vital role in the pathogenesis of depression. Our previous studies have found the disturbance of gut microbiota in depressed mice and MDD patients. ${ }^{16-18}$ Other researchers also reported the significant gut microbiome changes in MDD patients. ${ }^{41,42}$ Although these findings are not exactly the same, partly causing by the differences on inclusion/exclusion criteria of MDD patients, detection methods or analytical methods, the disturbance of phyla Firmicutes is viewed as the hallmark of depression. However, the molecular mechanisms of phyla Firmicutes in the onset of depression are still unclear. In this study, we found that the 14 of 17 differential genera belonged to phyla Firmicutes, and function prediction analysis showed that these differential genera were involved in four inflammationrelated pathways. Meanwhile, the four differential genera belonged to phyla Firmicutes were significantly correlated with the identified potential inflammation-related biomarkers. Taken together, we speculated that the disturbance of phyla Firmicutes might be involved in the onset of depression by regulating the host's inflammatory response.

The inflammatory response has a close relationship with many diseases. ${ }^{43-46}$ As the important part of immune system, it is viewed as a driving factor causing changes in neurocircuits and neurotransmitters, which are closely related to the onset of depressive symptoms. Mounting evidence has solidified the relationships between depression and inflammation. ${ }^{47,48}$ For example, a cumulative meta-analysis reported that the levels of $\mathrm{C}$-reactive protein and interleukin6 (IL-6) were higher in MDD patients than in non-depressed controls. ${ }^{49}$ Our previous studies found that the level of alpha 1 -antitrypsin (AAT) was decreased in MDD patients; ${ }^{27,50}$ AAT can inhibit the release of many pro-inflammatory factors, such as IL-6. In this study, we found ten inflammationrelated metabolites, and identified three inflammation-related pathways by analyzing these differential metabolites. Moreover, all the identified potential biomarkers were inflammation-related serum metabolites and significantly correlated with the disordered gut microbiota. These results suggested that it might be a good way to explore markers of inflammation for diagnosing MDD.

Several limitations of our study were as following: i) each group only had 60 participants, the relatively small number of samples indicated that our findings were needed future studies to validate and support; ii) all the participants were from the same place and with the same ethnicity, these similarities might limit the general applicability of our conclusion; iii) no single metabolomic platform had the ability to detect the entire metabonome in serum; thus, it was meaningful to use other metabolomic platforms to further study the differences of serum metabolites in MDD patients; iv) only MDD patients and HCs were recruited here to identify potential biomarkers for diagnosing MDD, future studies should explore whether our potential biomarkers could be used to separate MDD patients from patients with other psychiatric disorders; v) the two groups were matched for age, sex, and body mass index, but gut microbiota might also be influenced by other factors, such as dietary habits and smoke; thus, future studies should further explore the effects of these factors on our results; and vi) we did not know whether MDD patients or HCs received any nonpsychiatric medications; thus, future studies should investigate whether non-psychiatric medications could significantly affect our findings.

In conclusion, by conjoint analyzing the data of serum metabolites and gut microbiota compositions, we identified a potential serum inflammation-related biomarker panel for diagnosing MDD. The panel consisted of five inflammation-related metabolites: LysoPC(16:0), Deoxycholic acid, Docosahexaenoic acid, Taurocholic acid and LysoPC(20:0), which could effectively separate MDD patients from HCs. Our results provided candidates for future developing objective diagnostic methods for MDD, and showed novel insights for further exploring the role of gut microbiota in the pathogenesis of MDD.

\section{Acknowledgments}

This work was supported by the Natural Science Foundation Project of China (81701360, 81901398), the Science and Technology Fund of Guizhou Province [(QianHe LH2015)7411], the Open Project Funding of Shanghai Key Laboratory of Forensic Medicine (Academy of Forensic Science), the Natural Science Foundation of Chongqing, China (Grant No. cstc2019jcyjmsxmX0025), and the Chongqing Yuzhong District Science \& Technology Commission (20190115).

\section{Disclosure}

The authors declare that they have no conflicts of interest.

\section{References}

1. Frankish H, Boyce N, Horton R. Mental health for all: a global goal. Lancet. 2018;392(10157):1493-1494. doi:10.1016/S0140-6736(18) 32271-2

2. Liao D, Chen Y, Guo Y, et al. Salvianolic acid b improves chronic mild stress-induced depressive behaviors in rats: involvement of AMPK/ SIRT1 signaling pathway. $J$ Inflamm Res. 2020;13:195-206. doi:10.2147/JIR.S249363 
3. Wang HQ, Wang ZZ, Chen NH. The receptor hypothesis and the pathogenesis of depression: genetic bases and biological correlates. Pharmacol Res. 2021;167:105542. doi:10.1016/j.phrs.2021.105542

4. Beirão D, Monte H, Amaral M, Longras A, Matos C, Villas-Boas F. Depression in adolescence: a review. Middle East Curr Psychiatry. 2020;27:50. doi:10.1186/s43045-020-00050-z

5. Yano JM, Yu K, Donaldson GP, et al. Indigenous bacteria from the gut microbiota regulate host serotonin biosynthesis. Cell. 2015;161:264-276. doi:10.1016/j.cell.2015.02.047

6. Schwartz J, Murrough JW, Iosifescu DV. Ketamine for treatment-resistant depression: recent developments and clinical applications. Evid Based Ment Health. 2016;19(2):35-38. doi:10.1136/eb-2016-102355

7. Das R, Emon MPZ, Shahriar M, et al. Higher levels of serum IL-1 $\beta$ and TNF- $\alpha$ are associated with an increased probability of major depressive disorder. Psychiatry Res. 2021;295:113568. doi:10.1016/ j.psychres.2020.113568

8. Anjum S, Qusar MMAS, Shahriar M, et al. Altered serum interleukin-7 and interleukin-10 are associated with drug-free major depressive disorder. Ther Adv Psychopharmacol. 2020;10:2045125320916655. doi:10.1177/2045125320916655

9. Emon MPZ, Das R, Nishuty NL, et al. Reduced serum BDNF levels are associated with the increased risk for developing MDD: a case-control study with or without antidepressant therapy. BMC Res Notes. 2020;13(1):83. doi:10.1186/s13104-020-04952-3

10. Malik S, Singh R, Arora G, Dangol A, Goyal S. Biomarkers of major depressive disorder: knowing is half the battle. Clin Psychopharmacol Neurosci. 2021;19(1):12-25. doi:10.9758/cpn.2021.19.1.12

11. Bai S, Wang W, Wang $T$, et al. CD36 deficiency affects depressive-like behaviors possibly by modifying gut microbiota and the inflammasome pathway in mice. Transl Psychiatry. 2021;11 (1):16. doi:10.1038/s41398-020-01130-8

12. Fouquier J, Moreno Huizar N, Donnelly J, et al. The gut microbiome in autism: study-site effects and longitudinal analysis of behavior change. mSystems. 2021;6(2):e00848-20. doi:10.1128/mSystems.00848-20

13. Lee J, d'Aigle J, Atadja L, et al. Gut microbiota-derived short-chain fatty acids promote poststroke recovery in aged mice. Circ Res. 2020;127(4):453-465. doi:10.1161/CIRCRESAHA.119.316448

14. Lichota A, Gwozdzinski K, Szewczyk EM. Microbial modulation of coagulation disorders in venous thromboembolism. $J$ Inflamm Res. 2020;13:387-400. doi:10.2147/JIR.S258839

15. Zheng $\mathrm{P}$, Wu J, Zhang $\mathrm{H}$, et al. The gut microbiome modulates gut-brain axis glycerophospholipid metabolism in a region-specific manner in a nonhuman primate model of depression. Mol Psychiatry. 2020. doi:10.1038/s41380-020-0744-2

16. Wu M, Tian T, Mao Q, et al. Associations between disordered gut microbiota and changes of neurotransmitters and short-chain fatty acids in depressed mice. Transl Psychiatry. 2020;10(1):350. doi:10.1038/s41398-020-01038-3

17. Chen JJ, Zheng P, Liu YY, et al. Sex differences in gut microbiota in patients with major depressive disorder. Neuropsychiatr Dis Treat. 2018;14:647-655. doi:10.2147/NDT.S159322

18. Chen JJ, He S, Fang L, et al. Age-specific differential changes on gut microbiota composition in patients with major depressive disorder. Aging (Albany NY). 2020;12(3):2764-2776. doi:10.18632/aging.102775

19. Sanada K, Nakajima S, Kurokawa S, et al. Gut microbiota and major depressive disorder: a systematic review and meta-analysis. J Affect Disord. 2020;266:1-13. doi:10.1016/j.jad.2020.01.102

20. Lai WT, Deng WF, Xu SX, et al. Shotgun metagenomics reveals both taxonomic and tryptophan pathway differences of gut microbiota in major depressive disorder patients. Psychol Med. 2021;51(1):90-101. doi:10.1017/S0033291719003027

21. Pan X, Wen SW, Kaminga AC, Liu A. Gut metabolites and inflammation factors in non-alcoholic fatty liver disease: a systematic review and meta-analysis. Sci Rep. 2020;10(1):8848. doi:10.1038/ s41598-020-65051-8
22. Sae-Khow K, Charoensappakit A, Visitchanakun P, et al. Pathogenassociated molecules from gut translocation enhance severity of cecal ligation and puncture sepsis in iron-overload $\beta$-Thalassemia mice. J Inflamm Res. 2020;13:719-735. doi:10.2147/JIR.S273329

23. Leonard BE. Inflammation and depression: a causal or coincidental link to the pathophysiology? Acta Neuropsychiatr. 2018;30(1):1-16. doi:10.1017/neu.2016.69

24. Colasanto M, Madigan S, Korczak DJ. Depression and inflammation among children and adolescents: a meta-analysis. J Affect Disord. 2020;277:940-948. doi:10.1016/j.jad.2020.09.025

25. Zheng P, Wang Y, Chen L, et al. Identification and validation of urinary metabolite biomarkers for major depressive disorder. Mol Cell Proteomics. 2013;12(1):207-214. doi:10.1074/mcp.M112.021816

26. Zheng P, Gao HC, Qi ZG, et al. Peripheral metabolic abnormalities of lipids and amino acids implicated in increased risk of suicidal behavior in major depressive disorder. Metabolomics. 2013;9(3):688-696. doi:10.1007/s11306-012-0474-9

27. Bai S, Fang L, Xie J, Bai H, Wang W, Chen JJ. Potential biomarkers for diagnosing major depressive disorder patients with suicidal ideation. J Inflamm Res. 2021;14:495-503. doi:10.2147/JIR.S297930

28. Arentsen T, Qian Y, Gkotzis S, et al. The bacterial peptidoglycan-sensing molecule Pglyrp2 modulates brain development and behavior. Mol Psychiatry. 2017;22(2):257-266. doi:10.1038/mp.2016.182

29. Hoyles L, Snelling T, Umlai UK, et al. Microbiome-host systems interactions: protective effects of propionate upon the blood-brain barrier. Microbiome. 2018;6(1):55. doi:10.1186/s40168-018-0439-y

30. Chen L, Dong B, Gao H, et al. HPV-16 E2/E6 and POU5F1B as biomarkers to determine cervical high-grade squamous lesions and more. J Inflamm Res. 2020;13:813-821. doi:10.2147/JIR.S278911

31. Tang Y, Li Y, Sun J, Pan H, Yao F, Jiao X. Selection of an optimal combination panel to better triage COVID-19 hospitalized patients. J Inflamm Res. 2020;13:773-787. doi:10.2147/JIR.S273193

32. He S, Mao X, Lei H, et al. Peripheral blood inflammatory-immune cells as a predictor of infertility in women with polycystic ovary syndrome. J Inflamm Res. 2020;13:441-450. doi:10.2147/JIR.S260770

33. Liu Y, Duan Y, Li Y. Integrated gene expression profiling analysis reveals probable molecular mechanism and candidate biomarker in Anti-TNF $\alpha$ non-response IBD patients. $J$ Inflamm Res. 2020;13:81-95. doi:10.2147/JIR.S236262

34. Mandrekar JN. Receiver operating characteristic curve in diagnostic test assessment. $J$ Thorac Oncol. 2010;5(9):1315-1316. doi:10.1097/ JTO.0b013e3181ec173d

35. Wang S, Tang K, Lu Y, et al. Revealing the role of glycerophospholipid metabolism in asthma through plasma lipidomics. Clin Chim Acta. 2021;513:34-42. doi:10.1016/j.cca.2020.11.026

36. Chávez-Talavera O, Tailleux A, Lefebvre P, Staels B. Bile acid control of metabolism and inflammation in obesity, type 2 diabetes, dyslipidemia, and nonalcoholic fatty liver disease. Gastroenterology. 2017;152(7):1679-1694.e3. doi:10.1053/j.gastro.2017.01.055

37. Higgins AJ, Lees P. The acute inflammatory process, arachidonic acid metabolism and the mode of action of anti-inflammatory drugs. Equine Vet J. 1984;16(3):163-175. doi:10.1111/j.2042-3306.1984.tb01893.x

38. Liu $\mathrm{P}, \mathrm{Lu} \mathrm{Z}$, Liu $\mathrm{L}$, et al. NOD-like receptor signaling in inflammation-associated cancers: from functions to targeted therapies. Phytomedicine. 2019;64:152925. doi:10.1016/j.phymed.2019.152925

39. Zhou H, Urso CJ, Jadeja V. Saturated fatty acids in obesity-associated inflammation. $J$ Inflamm Res. 2020;13:1-14. doi:10.2147/JIR. S229691

40. Onomoto K, Onoguchi K, Yoneyama M. Regulation of RIG-I-like receptor-mediated signaling: interaction between host and viral factors. Cell Mol Immunol. 2021;18(3):539-555. doi:10.1038/ s41423-020-00602-7

41. Naseribafrouei A, Hestad K, Avershina E, et al. Correlation between the human fecal microbiota and depression. Neurogastroenterol Motil. 2014;26(8):1155-1162. doi:10.1111/nmo.12378 
42. Jiang $\mathrm{H}$, Ling $\mathrm{Z}$, Zhang $\mathrm{Y}$, et al. Altered fecal microbiota composition in patients with major depressive disorder. Brain Behav Immun. 2015;48:186-194. doi:10.1016/j.bbi.2015.03.016

43. Yang CC, Hsiao LD, Yang CM. Galangin inhibits LPS-induced MMP-9 expression via suppressing protein kinase-dependent AP-1 and FoxO1 activation in rat brain astrocytes. $J$ Inflamm Res. 2020;13:945-960. doi:10.2147/JIR.S276925

44. Lisowska B, Jakubiak J, Siewruk K, Sady M, Kosson D. Which idea is better with regard to immune response? Opioid anesthesia or opioid free anesthesia. $J$ Inflamm Res. 2020;13:859-869. doi:10.2147/JIR.S275986

45. Al Rushood M, Al-Eisa A, Al-Attiyah R. Serum and urine interleukin-6 and interleukin-8 levels do not differentiate acute pyelonephritis from lower urinary tract infections in children. $J$ Inflamm Res. 2020;13:789-797. doi:10.2147/JIR.S275570

46. Chang MX, Xiong F. Astaxanthin and its effects in inflammatory responses and inflammation-associated diseases: recent advances and future directions. Molecules. 2020;25(22):5342. doi:10.3390/ molecules 25225342
47. Felger JC. Role of inflammation in depression and treatment implications. Handb Exp Pharmacol. 2019;250:255-286. doi:10.1159/000343966

48. Raison CL, Rutherford RE, Woolwine BJ, et al. A randomized controlled trial of the tumor necrosis factor antagonist infliximab for treatment-resistant depression: the role of baseline inflammatory biomarkers. JAMA Psychiatry. 2013;70(1):31-41. doi:10.1001/2013. jamapsychiatry. 4

49. Haapakoski R, Mathieu J, Ebmeier KP, Alenius H, Kivimäki M. Cumulative meta-analysis of interleukins 6 and $1 \beta$, tumour necrosis factor $\alpha$ and C-reactive protein in patients with major depressive disorder. Brain Behav Immun. 2015;49:206-215. doi:10.1016/j. bbi.2015.06.001

50. Gui SW, Liu YY, Zhong XG, et al. Plasma disturbance of phospholipid metabolis in major depressive disorder by integration of proteomics and metabolomics. Neuropsychiatr Dis Treat. 2018;14:1451-1461. doi:10.2147/NDT.S164134
Journal of Inflammation Research

\section{Publish your work in this journal}

The Journal of Inflammation Research is an international, peerreviewed open-access journal that welcomes laboratory and clinical findings on the molecular basis, cell biology and pharmacology of inflammation including original research, reviews, symposium reports, hypothesis formation and commentaries on: acute/chronic inflammation; mediators of inflammation; cellular processes; molecular mechanisms; pharmacology and novel anti-inflammatory drugs; clinical conditions involving inflammation. The manuscript management system is completely online and includes a very quick and fair peerreview system. Visit http://www.dovepress.com/testimonials.php to read real quotes from published authors. 